\title{
Effects of Fibroblast Transplantation on the Content of Macrophages and the Morphology of Regenerating Ischemic Cutaneous Wounds
}

\author{
Yelena Yu. Shapovalova, PhD, ScD*; Tatyana A. Boyko, PhD; Yuriy G. Baranovskiy, PhD; \\ Marina N. Morozova, PhD, ScD; Nikolay P. Barsukov, PhD, ScD; \\ Fedor N. Ilchenko. PhD, ScD; Aleksey G. Baranovskiy, PGS \\ Medical Academy named after S.I. Georgievsky of Vernadsky CFU \\ Simferopol, the Russian Federation
}

\begin{abstract}
Background: The study of the morphological structure and the determination of macrophagal fraction (MF) in the newly formed epidermis and dermis on the 19th day after the transplantation of auto- and heterofibroblasts and a dermal equivalent with heterofibroblasts will allow determining the optimal method for ischemic wound healing.

Materials and Methods: The study was performed on 28 white mature mice of the C57/B1 line aged between 5 and 7 months. In an ischemic cutaneous wound, $0.4 \mathrm{ml}$ of fibroblast suspension ( 1.33 million cells) and a dermal equivalent were transplanted. The biopsy material was embedded in paraffin and stained with H\&E by the Weigert-Van Gieson method to visualize the elastic and collagen fibers. Macrophages were determined by monoclonal antibodies to CD68. On the 19th day of the healing of ischemic cutaneous wound, the wound healing process goes through the transition from the stage of proliferation with GT formation into the stage of differentiation or fibrosis. The most positive for regenerative histogenesis and inflammation is the introduction of autofibroblasts. The most differentiated epidermis is formed after transplantation into the wound of the dermal equivalent with heterofibroblasts due to the presence of hairpieces in the form of formed hair follicles. The favorable effect of the dermal equivalent with heterofibroblasts differs from the influence of the autofibroblast suspension only by several percent: the thickness of the epidermis by $4.29 \%$, the area of collagen fibers by $2.66 \%$, and the area of the blood vessels by $4.04 \%$. The most positive treatment for regenerative histogenesis and inflammation is the introduction of autofibroblasts. The most differentiated epidermis is formed after transplantation into the wound of the dermal equivalent with heterofibroblasts, due to the presence of pieces hair in the form of formed hair follicles.

Conclusion: The favorable effect of the dermal equivalent with heterofibroblasts differs from the influence of the autofibroblast suspension by only several percent: the thickness of the epidermis by $4.29 \%$, the area of collagen fibers by $2.66 \%$, and the area of the blood vessels by 4.04\%. (International Journal of Biomedicine. 2017;7(4):302-306.)
\end{abstract}

Key Words: ischemic cutaneous wound $\bullet$ regenerative histogenesis $\bullet$ fibroblast $\bullet$ dermal equivalent $\bullet$ macrophage

\section{Abbreviations}

EG, experimental group; GT, granulation tissue; H\&E, hematoxylin and eosin; MF, macrophagal fraction.

\section{Introduction}

A defect of the skin that persists for a long time against the background of reduced blood circulation remains a problem of modern surgery. ${ }^{(1)}$ There are various causes of

*Corresponding author: Prof. Yelena Shapovalova, PhD, ScD. Head of the Department of Histology \& Embryology of the Medical Academy named after S.I. Georgievsky of Vernadsky CFU. Simferopol, Russia.E-mail: Shapovalova_L@mail.ru loss of the skin, such as violation of the blood supply and innervation of the skin, trauma (including gunshot), local effects of high and low temperatures, ${ }^{(2)}$ ionizing radiation, and others. The problem of skin repair has still not been solved, although many methods have been proposed and applied, from pharmacological methods to surgical plastic closure of the wound. ${ }^{(3)}$ Currently, the world's achievements in molecular cell biology have created the basis for the application of cellular technologies in the treatment of long-term wound defects. ${ }^{(4)}$ One such treatment, now widely used, is the introduction of 
autologous and heterologous fibroblasts into the wound, which significantly shortens the healing time ${ }^{(5,6)}$ However, despite a number of works on this subject, ${ }^{(7,8)}$ the reorganization of the tissue components of the ischemic wound at different healing periods remains poorly understood.

The objective of our research was to study the morphological structure, collagen formation and angiogenesis in the biopsies of the newly formed epidermis and dermis on the 19th day of their recovery, in the ischemic wound model after the introduction of auto- and heterofibroblasts, and after dermal equivalent transplantation with heterofibroblasts.

\section{Materials and Methods}

\section{Design of the experiment}

The study was performed on 28 white mature mice of the C57/B1 line aged between 5 and 7 months. The animals were divided into the control group (CG), consisting of 7 individuals, and 3 experimental groups (EG1, EG2, and EG3) with 7 animals in each group. The animals were housed in keeping with the rules for good laboratory practice (GLP). The experiments were performed in accordance with the norms for the humane treatment of animals, which are regulated by Directive 86/609/EEC on the protection of animals used for experimental and other scientific purpose. In all groups, we performed an operation for modeling a cutaneous wound in the scapular region after intraperitoneal administration of a $2.5 \%$ solution of Avertin (0.3-0.4 ml). The skin was resected in the form of a circle with a diameter of $12 \mathrm{~mm}$. A silicone ring with an external diameter of $12 \mathrm{~mm}$ was fixed to the edges of the wound with a skin-fascial nodular suture with atraumatic suture material Polypropylene 5-0 to exclude the possibility of epithelialization of the wound and the closure of its mobile skin area ${ }^{(9)}$ The ischemia of the wound was carried out by superimposing the suture seam by use of Polypropylene 5-0 at a distance of $1.0 \mathrm{~cm}$ from the outer diameter of the wound, which disrupts blood circulation in the system near the scapular arteries. Arterial anastomosis around the scapula is formed by a. Circularisscapula (the branch of artery axillaris) and Ramus descendens (the branch of the transverse cervical artery originating from the Truncus thyrocervicalis). From the excised skin of the mice, fibroblasts were isolated under sterile box conditions with a laminar flow of air. After enzymatic removal of the epidermis, pieces of skin were placed in a DMEM-F12 medium (Lonza) and crushed with vascular scissors to a size of 1-2 $\mathrm{mm}$. Then equal volumes of solutions of type I collagenase $(200 \mathrm{U} / \mathrm{ml}$, Sigma) and dyspase (30 U/ $\mathrm{ml}$ ) (Gibco) were added to the pieces of tissue. The resulting mixture was incubated for 1 hour at $37^{\circ} \mathrm{C}$ with constant stirring. After filtering the suspension through a $0.40 \mu \mathrm{m}$ diameter filter and centrifuging for 7 minutes at $1000 \mathrm{rpm}$, the fibroblasts were re-suspended and cultured in the DMEM-F12 medium supplemented with $10 \%$ calf serum (HyClone) and $50 \mathrm{U} / \mathrm{ml}$ penicillin-streptomycin (PanEco) in Petri dishes. The Petri dishes were placed in an incubator at $37^{\circ} \mathrm{C}$ and $5 \% \mathrm{CO}_{2}$ to reach $100 \%$ confluence. Trypsin( $(0.25 \%)-\operatorname{EDTA}(0.02 \%)$ solution was used to transfer the cells.

In EG1 and EG2, $0.4 \mathrm{ml}$ of fibroblast suspension of the first or second passage in the growth medium DMEM-F12 in the amount of 1.33 million cells was intraoperatively injected into the bottom of the wound and around it. In EG1, we used heterofibroblasts, in EG2 - autofibroblasts. In EG3, a dermal equivalent with heterofibroblasts, prepared on the bases of type I collagen from rat tails, was transplanted into the wound. A sterile $0.34 \mathrm{M}$ solution of $\mathrm{NaOH}$ was combined with a concentrated (x10) Medium 199 in a 1:1 ratio. The resulting mixture was combined with a cooled solution of collagen, after which a suspension of fibroblasts was added to the DMEM-F12 culture medium, containing $10 \%$ fetal serum (HyClone). The resulting mixture was incubated at $37^{\circ} \mathrm{C}$ until the gel was completely polymerized. ${ }^{(10)}$

\section{Morphological examination of scars}

On the 19th day after the operation, in all groups, the formed scar was intraoperatively excised and fixed in a $10 \%$ buffered formalin solution for morphological examination. The material was embedded in paraffin and stained with H\&E and by the Weigert-Van Gieson method to visualize the elastic and collagen fibers. Morphological examination of histological preparations was carried out with a OLIMPUS SX-31 light-optical microscope. The thickness of the epidermis, the number of microvessels in the sections, the area of collagen fibers and microvessels in the dermis of the scars were determined using an image analysis program (Image J 1.46r, National Institutes of Health, USA). We use a 10X ocular lens and a 40X objective lens (a total magnification of 400X). Fifty measurements were performed in each group. The obtained digital data (expressed in pixels) were converted into $\mu \mathrm{m}$ by using special coefficients: 6379251 for a $10 \mathrm{X}$ lens and 98911797 for a 40X lens.

Immunohistochemical identification of macrophages

The presence of macrophages was determined by an immunohistochemical method after dewaxing and rehydrating paraffin sections. To restore the antigenic properties of the cells of the regenerate tissue after fixation in formalin, heat-induced epitope retrieval was performed. The primary antibodies were CD68-polyclonal antibodies (Gene Tex Inc., USA) in a 1:100 dilution. Secondary antibodies containing a large number of horseradish peroxidase molecules were applied to histological sections and incubated in a humid chamber for 30 minutes with washing in Tris-buffer solution between each stage for 10 minutes. To detect and visualize the reaction, from 1 to 3 drops of 3,3-diaminobenzidine (DAB Chromogen/Substrate) (Gene Tex, USA) were added to each section. To adequately represent the structure of the tissue and cell nuclei, the sections were additionally stained with Meyer's hematoxylin for 3 minutes. The sections were dehydrated and placed in Aquatex gel (aqueous mounting agent; Andwin Scientific, France) under cover glasses. In addition, a control study was performed to exclude pseudo-positive and pseudo-negative results.

Macrophagal fraction (MF) was determined by counting the number of CD68-positive cells per 100 cells during microscopic examination (x1350 magnification), followed by calculating the average percentage, based on the results of the studied sections of each biopsy specimen in CG and EGs.

Statistical analysis was performed using the statistical software «Statistica». (v6.0, StatSoft, USA) and Microsoft 
Excel 2007. Baseline characteristics were summarized as frequencies and percentages for categorical variables and as mean \pm SEM for continuous variables. The Mann-Whitney (U Test) was used to compare the differences between the two independent groups. A probability value of $\mathrm{P}=0.05$ was considered statistically significant.

\section{Results}

During the healing of an ischemic cutaneous wound, the silicone ring fell away spontaneously due to the gradual epithelialization of the wound from the edges to the center and the eruption of the ring-retaining seams, which was regarded as an important sign that the regenerative processes were active. In CG, the silicone ring fell away on day $12.4 \pm 0.10$ after the operation to create an ischemic cutaneous wound. Under the thick remains of the scab, we found the complete wound epithelialization. In CG, on the 19th day after the operation, the epidermis of the biopsy specimen was formed by a multilayer epithelium with a thickness of $55.24 \pm 0.11 \mu \mathrm{m}$. We found a basal layer and several rows of spiny cells; a granular layer was fragmentarily visible. The stratum corneum was thin and at the initial stages of cell differentiation (Fig.1).

In EG1, epithelialization of the wound and falling away of the silicone ring took place one day earlier than in $C G$, namely, on day $11.4 \pm 0.06$ after the operation and transplantation of the suspension of heterofibroblasts in growth medium DMEM-F12. The thickness of the epidermis $(64.29 \pm 0.20 \mu \mathrm{m})$ was higher by $14.08 \%(\mathrm{P}=0.05)$ than in CG. The epidermis was formed and consisted of four layers: basal, prickly, granular and horny. The granular layer was seen by sections.

In EG2, epithelialization of the wound and falling away of the silicone ring were recorded even earlier than in CG and EG1 - by day $11.00 \pm 0.01$ after operation. On the 19th day of regenerative histogenesis, the tissue defect of the skin was eliminated most significantly. The thickness of the epidermis was higher than in CG by $50.52 \%(\mathrm{P}=0.05)$. Layers of the epidermis were significantly more differentiated. A pronounced stratum corneum was visible on the surface. The epidermis extended into the underlying granulation tissue (GT), forming papillae and hair follicles.

In EG3, the silicone ring fell away on day $12.20 \pm 0.11$ after the operation. On the 19th day after transplantation of the dermal equivalent with heterofibroblasts, the wound was covered with a thick layer of epidermis. The epidermis looked more differentiated than in the previous groups. All layers of the epidermis were present and well developed, including the granular layer. The thickness of the epidermis was $102.74 \pm 1.13 \mu \mathrm{m}$, which was greater by $43.87 \%(\mathrm{P}=0.05)$ than in $\mathrm{CG}$. The epidermis formed the outgrowths into the underlying GT, which were the basic islets for hair development and formation of the papillary layer of the dermis.

In the biopsy specimens of CG and three EGs, under the epidermis there was GT filling the wound cavity. In CG, the boundary between the epidermis and the future dermis was clear, but no full papillae were formed. GT was formed by interlacing collagen fibers without a clear orientation, between which there were cells represented mainly by functionally active fibroblasts. Collagen fibers occupied $33.76 \pm 0.22 \%$ of the dermis area. We did not find elastic fibers. A few blood capillaries and venules were widened, and their area constituted $1.02 \pm 0.01 \%$ of the dermis area. Vertically arranged capillaries of various diameters were identified. The internal surface of the vessels was lined with a single layer of endothelial cells. CD68-positive cells, which are identified as macrophages, were localized mainly around blood vessels or in the lumen of the vessels, which confirms their hematogenous origin (Fig. 2).

In EG1, GT did not differ morphologically from control, but collagen fibers occupied an average of $55.44 \pm 0.17 \%$ of the dermis area. CD68-positive cells colored in brown were present near the blood vessels. In EG2, GT was characterized by a significant increase in the processes of angiogenesis and collagen formation. The collagen fibers acquired a regular orientation parallel to the epidermis, especially noticeable in the deep layers; their area was $63.14 \pm 0.12 \%$ of GT area, and the area of vessels was $1.73 \pm 0.01 \%$. (Fig. 3) We did not find leukocyte infiltration, but we did find a small number of macrophages in the deep layers of the dermis (Fig.4). Cellular elements of the fibroblastic series were represented by the large and elongated adventitious cells, which indicated their functional activity. Elastic fibers were absent in all parts of the dermis. In EG3, we found the formed hair tabs in GT of the dermis. The area occupied by collagen fibers was $60.15+0.37 \%$, and the area of the vessels was $1.68 \pm 0.01 \%$ of GT area. Thin and weakly oriented bundles of collagen fibers filled out the entire dermis. We found a small amount of CD68-positive cells near the blood vessels. Table 1 shows the change in the area of vessels, collagen fibers, and MF in GT of skin biopsy specimens in CG and EGs.

Table 1.

Change in the area of vessels, collagen fibers, and MF in GT of skin biopsy specimens in $C G$ and $E G S$

\begin{tabular}{|c|c|c|c|c|}
\hline Group & $\begin{array}{c}\text { Change in } \\
\text { the area of ves- } \\
\text { sels by relative } \\
\text { to CG, in \% }\end{array}$ & $\begin{array}{c}\text { Change in the } \\
\text { fibers relative } \\
\text { to CG, in \% }\end{array}$ & MF & $\begin{array}{c}\text { Change in MF } \\
\text { relative to CG, } \\
\text { in \% }\end{array}$ \\
\hline CG & 0 & 0 & $17.21 \pm 0.02$ & 0 \\
\hline EG1 & +14.29 & +39.11 & $15.14 \pm 0.02$ & -10.03 \\
\hline EG2 & +43.33 & +46.53 & $9.75 \pm 0.03$ & -43.35 \\
\hline EG3 & +39.29 & +43.87 & $10.66 \pm 0.02$ & -38.06 \\
\hline
\end{tabular}

Thus, angiogenesis was most active against the background of autofibroblast transplantation, when the area occupied by the vessels in the dermis is the largest and the percentage of its growth in comparison with CG is maximal. In this case, collagen formation was also most active. At the same time, after transplanting the dermal equivalent with heterofibroblasts into the experimental wound, we observed a pronounced favorable course of the wound process: the percentage increase in the area of the vessels and collagen fibers was less than in EG2 by only $2.66 \%$ and $4.04 \%$, respectively, and epidermal differentiation was most advanced. MF was the smallest in EG2 and this parameter was lower than in CG. 


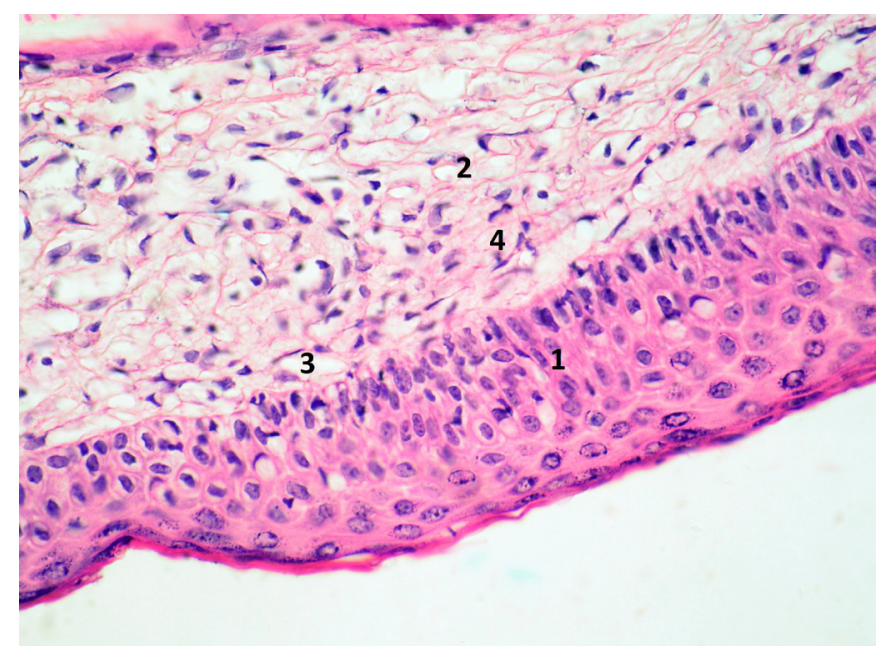

Fig. 1. Mouse skin biopsy. Control group. $H \& E$ staining. 1 - epidermis; 2 - granulation tissue; 3 - vessel; 4 - collagen fibers. Magnification: $x 200$

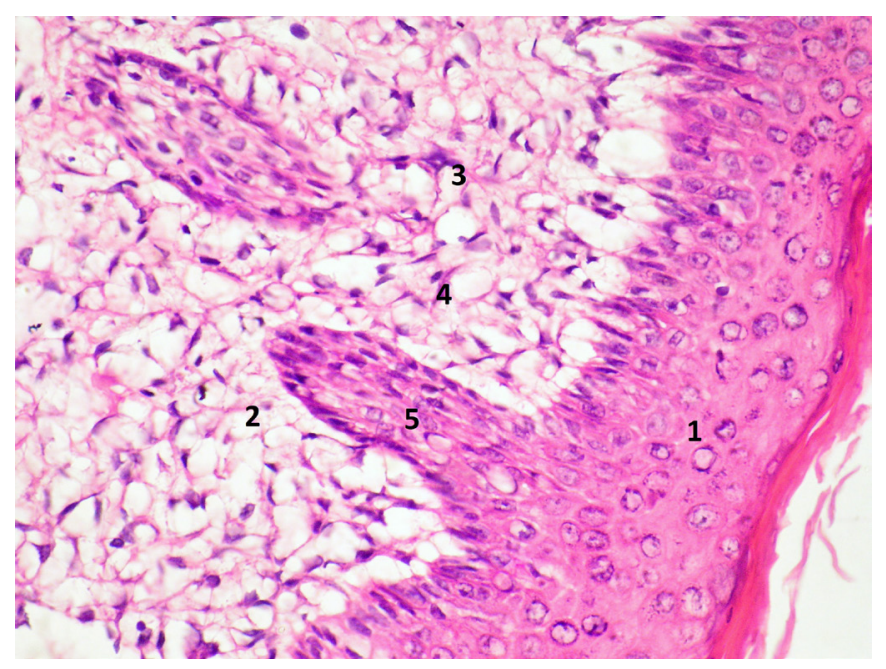

Fig. 3. Mouse skin biopsy (EG2): after autofibroblast transplantation. H\&E staining. 1 - epidermis; 2 granulation tissue; 3 - vessel; 4 - collagen fibers. Magnification: $x 200$.

\section{Discussion}

The process of skin wound healing includes three stages: inflammation, proliferation with GT formation, and remodeling or fibrosis. ${ }^{(1)}$ With the normal healing of wounds, an influx of inflammatory cells to the wound site occurs until the fourth to sixth day, followed by a proliferative phase, during which the inflammatory cells are replaced by fibroblasts. In long-term, non-healing ischemic wounds, the elements of all three phases can simultaneously be present, but inflammation is predominant. ${ }^{(12)}$ Reduction of inflammation promotes an activation of GT development with subsequent fibrosis. Macrophages can serve as a marker of inflammatory activity due to their ability to excrete pro-inflammatory cytokines. A low MF in GT indicates the abatement of the inflammatory phase of wound repair and the active formation of GT, a

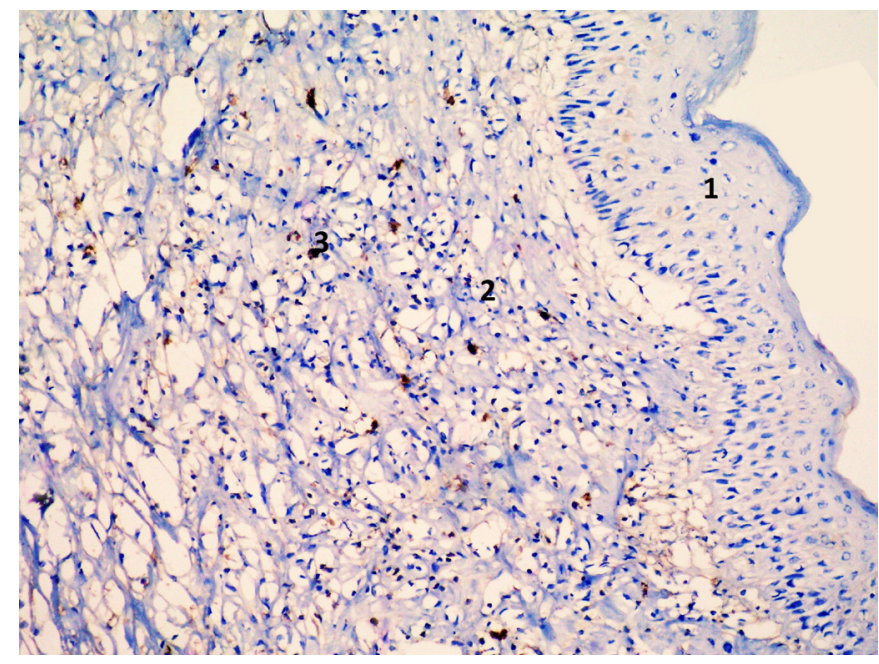

Fig. 2. Mouse skin biopsy. Control group. Immunohistochemical identification of macrophages. 1 -epidermis; 2 - granulation tissue; 3 - CD68-positive cell (macrophage). Magnification: $x 100$.

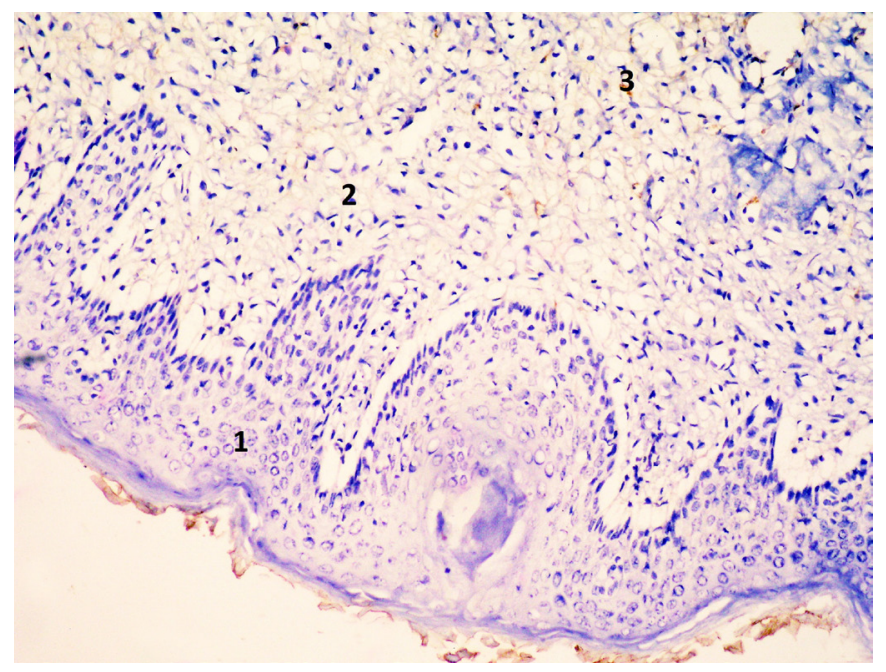

Fig. 4. Mouse skin biopsy (EG2): after autofibroblast transplantation. Immunohistochemical identification of macrophages. 1 - epidermis; 2 - granulation tissue; 3 CD68-positive cell (macrophage). Magnification: $x 100$.

temporary matrix, which performs mechanical and regulatory functions. In the search for approaches to reducing the time of inflammation, it was shown that dermal fibroblasts are the source of adiponectin, which acts as an active antiinflammatory cytokine and induces the production of antiinflammatory factors such as IL-10 and IL-1RA. ${ }^{(13)}$ According to our data, autofibroblasts have the most pronounced antiinflammatory effect, which significantly shortens the healing time of a cutaneous ischemic wound.

\section{Conclusion}

Thus, on the 19th day of the healing of an ischemic cutaneous wound, the wound healing process goes through the transition from the stage of proliferation with GT formation into the stage of differentiation or fibrosis. The most positive 
treatment for regenerative histogenesis and inflammation is the introduction of autofibroblasts. The most differentiated epidermis is formed after transplantation into the wound of the dermal equivalent with heterofibroblasts, due to the presence of pieces hair in the form of formed hair follicles. The favorable effect of the dermal equivalent with heterofibroblasts differs from the influence of the autofibroblast suspension by only several percent: the thickness of the epidermis by $4.29 \%$, the area of collagen fibers by $2.66 \%$, and the area of the blood vessels by $4.04 \%$.

\section{Competing interests}

The authors declare that they have no competing interests.

\section{References}

1. Govrin G. New method for treating hard-to-heal wounds: clinical experience with charged polystyrene microsphers. Wounds. 2010;6(4):52-61.

2. Danilov RK. Wound process:Histogenetic basis. SPb.: VMedA; 2008: 380 pp. [in Russian].

3. Khrupkin VI, Zubritskiy VF, Ivashkin AN. Dermatoplasty of wound defects. M: GEOTAR-Media; 2009: 102 pp. [in Russian].

4. Vinnik YuS, Salmina AB, Drobushevskaya AI. Cellular technologies and tissue engineering in the treatment of longterm healing wounds. Vestnik eksperimental'noy i klinicheskoy khirurgii. 2011;4(2):392-397. [Article in Russian].

5. Shapovalova EYu, Boyko TA, Baranovskiy YuG, Kharchenko SV., Yunsi GA. Morphological characteristics of the healing of an ischemic experimental wound on the 12th day after application of auto- and hetero-fibroblasts and dermal equivalent. Mezhdunarodnyy nauchno-issledovatel'skiy zhurnal. 2017;62(8-3):151-5. [Article in Russian].

6. Porter S. The role of the fibroblast in wound contraction and healing. Wounds. 2007;3(2):45-48.

7. Zorin VL, Zorina AI, Petrakova OS, Cherkasov VR. Dermal fibroblasts for the treatment of skin defects. Geny \& Kletki. 2009; 4(4): 26-40. [Article in Russian].

8. Meleshina AV, Bystrova AS, Rogovaya OS, Vorotelyak EA, Vasil'ev AV, Zagaynova EV. Tissue engineering skin constructs and the use of stem cells to create skin equivalents (review). Sovremennye tekhnologii v meditsine. 2017;9(1):198-218. [Article in Russian].

9. Baranovskiy YuG, Ilchenko FN., Shapovalova EYu. Method for modeling trophic ulcers in laboratory mice in the experimental model. Vestnik neotlozhnoy i vosstanovitel'noyk hirurgii. 2016; 1 (2): 259-261. [Article in Russian].

10. Andreev DYu, Abramova NV, Blinova MI, Pinaev GP. Effectiveness of cutaneous plastics and dermal equivalent in the treatment of extensive ulcers of the tibia of mixed origin. Vestnik khirurgii im. I.I. Grekova. 2013;172 (1): 104-107. [Article in Russian].

11. Dong X, Mao S, Wen H. Upregulation of proinflammatory genes in skin lesions may be the cause of keloid formation (Review). Biomed Rep. 2013;1(6):833-836.

12. Vorotnikov AV, Suzdal'cova JuG, Rubcov JuP, Aniol NV, Gorjunov KV, Kudrjashova TV, et al. Directed migration and mesenchymal progenitor cells: participation in inflammation, repair and tissue regeneration. Stvolovye kletki i regenerativnaja medicina: Sbornik statej. M: MAKS Press. 2012: 62-97. [Article in Russian].

13. Esfahani M, Movahedian A, Baranchi M, Goodarzi MT.Adiponectin: an adipokine with protective features against metabolic syndrome. Iran J Basic Med Sci. 2015;18(5):430-42. 